\title{
The "Wellbeing Check-In" Service Establishing, Developing and Tailoring a New Program to offer a Student-centred Response to COVID-19
}

\author{
Sarah Power \\ Student Health and Wellbeing Projects Coordinator \\ Georgina Hanna \\ Senior Health and Wellbeing Coordinator - Complex Case Manager \\ La Trobe University, Australia
}

\begin{abstract}
The Wellbeing Check-In service was developed as a tailored service to support the wellbeing of our students during the COVID-19 pandemic. Knowing that our students would require additional forms of support during the unprecedented times ahead, we carefully designed the service to be aligned with the new online access requirements. The original premise of the service was for students to "request a call" from the team, in order to provide bespoke, student-centred support for each student's situation. The service saw an immediate and unexpected uptake from both the students themselves and through referrals from a wide range university areas and staff, that has continued through the four months that the Wellbeing Check-In service has been operational. While the COVID-19 pandemic continues, we anticipate that the demand for this service and its style of support will only increase.
\end{abstract}

\section{Keywords}

Wellbeing, Student Wellbeing, Check-In, COVID-19, pandemic, COVID-19 response, support, access, online, mental health, anxiety, stress, tertiary students

\section{Background}

The Student Health and Wellbeing (SHW) portfolio is responsible for the delivery of holistic wellbeing services to La Trobe University (LTU) students with the core functions ranging across a spectrum from prevention to reactive front-line services. These include Counselling Services, the AccessAbility Hub (including Inclusive Resource Development), Safer Community, behavioural management support, threat assessment, specialised wellbeing services as well as preventative, educative and health promotion initiatives and activity.

With the increasing concerns caused by the spread of COVID-19 throughout the world and into our communities, this focus became even more critical.

With escalating community fears, we anticipated that there would be practical day-to-day impacts on our students' lives brought about by COVID-19. We were also concerned about these impacts effecting their mental health and wellbeing. Looming pandemic protocols and ongoing government mandated isolation, social distancing measures and the widespread move to working-from-home, all added to the impact of secondary stressors such as escalated financial and housing concerns. We knew that students would require additional support from our existing services, beyond simply moving these supports online.

Our team designed and implemented a new service that we called "Wellbeing Check-Ins", in order to increase the accessibility and visibility of SHW services. Given the "new normal" of the COVID19 world would likely mean our students would require support beyond the current service parameters, we hoped this new service would allow students to engage with SHW in a more holistic manner, and break down some of the stigma that may prevent them seeking help. 


\section{Design and description of the program}

\section{Initial crisis response and program conception}

During the unfolding of "the new COVID-19 world" the Senior Executive Group wanted LTU's response to the escalating situation to actively consider student wellbeing. Because of this, the SHW team was incorporated into the university's broader critical incident planning from the very beginning.

In the initial few days, the response of the SHW team was necessarily reactive. The SHW team's Complex Case Manager (CCM) was named as the key contact person and referral point for concerns regarding the wellbeing of students exposed to COVID-19. This proactive outreach was coordinated by the Portfolio Director in response to individual and groups of students known to have been exposed to, or diagnosed with, COVID-19. Additionally, the CCM also became the referral point for domestic and international students who were overseas and had difficulty returning to Australia for study as a result of travel restrictions put in place because of COVID-19. This was in response to these students contacting lecturers, coordinators, and various other university teams such as Accommodation and La Trobe International for help.

The nature of the service provided meant that a response needed to be flexible and, in many instances, "time critical". However, it quickly became clear that the number of referrals and contacts made in these early days would be unsustainable long term, at least in this initial format and delivery. Given the complex situations students were facing as a result of the pandemic, a practical, more efficient framework needed to be developed for SHW to continue to offer a responsive, high quality service throughout the duration of the pandemic. These considerations led to the conception and initiation of the "Wellbeing Check-In" service.

\section{Program installation and accessibility}

Considering the impending nationwide university campus shutdowns, we began the initiative by considering the most accessible way that students could contact the program we were designing. Our team worked with the LTU's "pause" to the semester and the upcoming broader shift to online learning and designed the service's accessibility around a web-based platform. To facilitate this, two SHW Project Coordinators collaborated with the LTU's digital marketing team to design the new pages and the associated form for students to request contact. They then incorporated these into the already existing SHW hub website.

The design of the self-referral request form was kept as simple and concise as possible for students. This allowed us to streamline the processes required for staff to respond and ensured that our studentcentred approach was kept paramount. The form asks students to provide basic demographic and contact information, as well as an invitation to outline the "key" issues of concern that prompted them to contact the service.

The Project Coordinators chose careful phrasing for the request form and its introduction. They avoided clinical terms like "appointment" and "counselling" to ensure that the range of concerns students could discuss remained broad. Careful thought and consideration went into the simpler elements of the pages, such as the images attached to each webpage tile and the order the tiles appeared in. The intention was to make the service friendly, approachable and accessible.

Consideration was also given to where, and how best, the Wellbeing Check-In service would be built into the website. The service was installed on the primary SHW hub page as a banner. Part of a decorative heading, and pinned to the top of the webpage, this installation makes the Wellbeing Check-In service highly visible, and the first thing students see when they open the SHW hub webpage. This made it easy to direct students to it as a resource point, or for students to independently locate and use the online portal to reach out for help. 
Our Project Coordinators have continued to build and improve the COVID-19 webpage, presenting further resources and supportive information SHW created collaboratively, as the need for them developed.

\section{Program staffing}

The Wellbeing Check-In service is coordinated by the CCM. The CCM's program, Wellbeing CONNECT, is a relatively new program within the SHW suite of services. The primary function of the CCM's role is the provision of intensive support to students who present with multiple psychosocial, risk, behavioural and/or health issues that impact on the success of academic, psychological and social pursuits. This was fully compatible with the desired functions of the new initiative and made the CCM best suited to support the complex nature of the impact of COVID-19. The responsive, time critical reactions that the initiative demanded (with the same, if not increased, complexity encountered in students' presenting issues) fitted well within the scope of the CCM's standard, student-centred practice.

While on paper, Wellbeing CONNECT has effectively been "paused" in order to respond to the enthusiastic uptake of the Wellbeing Check-In service, several students are still receiving on-going support from the program. Already a vulnerable cohort, these students' situations were only exacerbated by COVID-19 and the CCM found that they needed more support, not less, during this time. The CCM continues to accept referrals and the Wellbeing CONNECT service remains ongoing in the background as required.

A Project Coordinator, also an existing staff member within the Wellbeing unit, assists the Wellbeing Check-In team, by coordinating program support, record keeping and data analysis for the program.

In addition to the CCM, the Wellbeing Check-In team is supported by two Wellbeing Coordinators, on a fractional part-time basis. One of these Coordinators is an existing staff member within the SHW team, who possessed the required skill set to provide direct student support, as required. The other Coordinator, who was allocated to the team (two months into operation) is a registered psychologist who supports the Wellbeing Check-In program on a casual basis.

As the Wellbeing Check-In service is staffed by team members already active in other roles within SHW, there was no requirement for budgetary approval and only minimal outlay to initiate the service. Similarly, due to the scope of the roles co-opted to the new program, there was minimal training required for the staff beyond operational guidelines specific to the initiative. This meant we were able to begin this new initiative quickly, unrestricted by budget or staff recruitment, ${ }^{1}$ becoming fully operational in a matter of days.

\section{Scope and operational framework}

The framework of this initiative was developed with an understanding of the operational scope and limitations of other units within the SHW team. The design of this program complements the existing services but does not act as a replacement for them.

The Wellbeing Check-In service is not intended to be a crisis response service, nor does it provide comprehensive mental health assessment and/or diagnosis. Furthermore, it does not provide medical advice for COVID-19 related concerns or legal advice on government pandemic protocols.

The service design offers students an opportunity to "request a call" for a Wellbeing Coordinator to "check in" on their wellbeing, starting with the key issues identified on the student's submitted form.

In addition to the primary "self-referral" pathway, staff across the university (from within the SHW team and the broader university community) can refer students to the Wellbeing Check-In service for a proactive call. These referrals are based primarily on the following three scenarios:

\footnotetext{
${ }^{1}$ Two months into operation, additional casual budget was allocated to the program which allowed for the hire of our casual Coordinator and helped the team to manage student demand.
} 
1. Students with a confirmed diagnosis of COVID-19 and those self-isolating due to exposure to a confirmed case (either on campus or via student placement) are referred to the service for a proactive, outreach call;

2. Students who have expressed a level of psycho-social stress in their call from the Student Success team during their outbound call campaign ${ }^{2}$ were streamlined through the Success team leader and referred to the service to check on the students' wellbeing; and

3. Students who provided concerning personal statements in their financial bursary application were also referred to the service for outreach and provision of information.

Careful work was put into communications sent to the university community, advertising how to access the self-referral pathway. This aimed to increase general awareness of the Wellbeing CheckIn service and encourage self-referral. This communication was also used to remind the broader university community of the standard consultation pathways within SHW that remained available for referrals and support as normal.

Specific communications proactively went to students who were identified as being exposed to or diagnosed with COVID-19 during their study or placement. The CCM was explicitly identified by name, and her mobile work number was provided to these students as an available support during a period of often heightened anxiety and stress.

The development of the Wellbeing Check-In service protocols, and the formalisation of referral pathways for students exposed to potential COVID-19 infection, or students who tested positive meant that the team was able to move from reactive, "crisis", time-critical responses in these cases, to a more structured, streamlined, and sustainable process. This allowed risk to be better managed and provided this cohort more comprehensive wellbeing support.

Both self-referrals and referrals from staff are triaged for urgency, and in accordance with the issues identified in the referral. If the referral or request for a check-in clearly falls within the scope of another unit of the SHW team, the student is appropriately referred to ensure they receive the most appropriate support. For example:

- Where significant mental health concerns (including self-harm or harm to others) are indicated in the referral, the referral is escalated to the counselling team in a time critical manner;

- Regional students were referred to, and then coordinated by, the regional counselling manager and wellbeing teams, as those practitioners have greater local knowledge and expertise; and

- Recently, the Wellbeing Check-In team have also been asked to provide assessment support for an additional financial aid program, "Exceptional Emergency Aid", designed for students in most need. While not the focus of this article, or the Check-In program, this additional initiative is worthy of mention as it is an emerging task added to the scope of this team.

\section{Clinical tools and developed assessment templates}

Students are called by a member of the Wellbeing Check-In team on their specified contact number for discussion regarding their identified concerns. During the call, the Coordinators primarily use two templates developed by the CCM, in order to facilitate comprehensive assessment of the students' wellbeing.

The first tool is a psychological first aid template, informed by and based on the action principles of psychosocial first aid, used "when people are presented with community level crisis situations that

\footnotetext{
${ }^{2}$ This campaign is described in more detail in the "Challenges" Section
} 
could induce feelings of fear, risk of injury or death, or cause people intense feelings of distress" (Australian Red Cross \& Australian Psychological Society, 2020, p. 9).

The first questions on the template prompt the recording of demographic information with the student, including their source of referral to the Wellbeing Check-In service and a privacy statement to meet requirements of risk assessment, record keeping and privacy obligations. The "Background" section enables the Coordinators to begin to build some rapport with the student.

The template then moves through a series of questions designed to assist staff in developing a more complete understanding of the student's situation, centred on the five elements of psychosocial first aid; "ensuring safety", "promoting calm", "promoting connectedness", "promoting self-efficacy", and "instilling hope" (Australian Red Cross \& Australian Psychological Society, 2020, p18). This format allows the Wellbeing Check-In Coordinator to create space for an open conversation to explore these elements with the student, supporting our desired intervention of "a humane supportive response to a fellow human being who is suffering or may need support" (Australian Red Cross \& Australian Psychological Society, 2020, p. 15).

The second template is based on a comprehensive Psychosocial Assessment framework. Psychosocial assessments:

are a primary practice tool of the social work profession... [the tool] explore(s) the physical, psychological and social aspects of the client and their situation. This includes problems and strengths in social role functioning; in meeting financial and other basic needs; in family interactions/significant relationships and other social supports; and cultural factors. (Australian Association of Social Workers, 2015, p.3)

This template begins with some prompting questions that assist introductions and encourage rapportbuilding. The template offers some general "tips" in sourcing referral options, and gathers demographic and contextual information, with the purpose of identifying what the student is currently facing. A privacy statement is also included for discussion with the student.

Following this, several broad headings are highlighted to explore with the student, including:

- Home/Environment/Living;

- Education/Employment, Finances;

- Social Connections;

- Activities/Adjustment to C19 and or Current Situation;

- Mood/Mental health; and

- Supports/Services/Links (if any)

Spaces are provided for the Coordinator to write an assessment/summary of their conversation with the student, summarise any actions that will follow and outline a proactive plan to move forward.

Both templates have only been provided to our staff as a guide. There remains a need for a high level of assessment and clinical skill from the Wellbeing Coordinator in order to gauge and negotiate whether the student is open to explore their situation comprehensively via one of these frameworks, whether the student required a less "structured" approach, or whether the student prefers to focus on a single or specific issue that is causing them concern.

Our team members also have on hand the Mini Mental State Examination (MSE) (Folstein, Folstein, \& McHugh, 1975), and the MSE Rapid Recording form (Patrick, 2000) should they observe any concerning behaviours, speech patterns or perceptions. However, the Wellbeing Check-In service is not advertised as, and does not act as, a mental health crisis line, nor offer mental health assessment 
or diagnosis. Should students display any of these flagged issues, the student is referred to the Counselling Service Manager for further mental health assessment.

As we continued to work with students, it became apparent that some students required follow-up support and short-term case management to ensure best outcomes, assist with multiple issues, and keep our student-centred focus. A key intervention facilitated by the Wellbeing Check-In team has been the provision of support for the student and the enabling of resource-seeking by the student. In many cases, internal and external referrals to other services have also been provided. The Wellbeing Check-In team has made a concerted effort where possible to minimise the student feeling like they were being "shuffled around". At times, however, Coordinators have needed to balance this requirement with encouraging the students' independence, asking them to seek or access the newly found resources autonomously.

\section{Planned program evaluation}

Due to the current workloads across the team, there has been no program evaluation to date, other than the continued uptake and increase in service usage.

Because of privacy restrictions and the Health Records Act, gathering evaluations of the program from the participating students may be difficult and would need to be carefully negotiated. There are tentative plans to utilise anonymous survey platforms such as SurveyMonkey to gain feedback from a sample of students that have utilised the service. Careful consideration is currently being given to the viability of surveying students who were referred to the service for an outreach call rather than self-referred.

Feedback will also be sought from staff members who have referred students to the service, however this will be limited by our inability to provide outcome information on students they referred. This remains in the planning phase at present, as the Wellbeing Check-In team remains actively responding to a high volume of requests and referrals, reliant on time-critical action.

\section{Outcomes}

While we recognise that formalized program evaluation is ideal, given that the service is still active, and given our adherence to privacy mandates, this is not possible at this time.

Despite this, we feel that associated program statistics (below) can be used as an intermediate measure to analyse service outcomes until such evaluations can occur.

\section{Website portal access and traffic}

We ran Adobe Analytics, on the SHW hub webpage, to demonstrate the popularity and access to the developed program and its associated resources. The data are presented in Table 1.

Table 1 Rank and Percentage of Total Clicks on SHW Service Webpages

\begin{tabular}{|c|c|c|c|c|}
\hline \multirow[b]{2}{*}{ Service } & \multicolumn{2}{|c|}{ Clicks in the Past Year } & \multicolumn{2}{|c|}{ Clicks Since Wellbeing } \\
\hline & Rank & $\%$ & Rank & $\%$ \\
\hline Wellbeing Check-In & 2 & 17.60 & 1 & 33.47 \\
\hline Counselling and mental health & 1 & 18.47 & 2 & 11.46 \\
\hline Equity \& Diversity & 3 & 9.87 & 3 & 6.37 \\
\hline
\end{tabular}

Note. The date range for "Clicks in the Past Year" is July $23^{\text {rd }}, 2019-$ July $23^{\text {rd }}, 2020$. The date range for "Clicks Since Wellbeing Check-In Began” is March 23 ${ }^{\text {rd }}, 2020$ - July $23^{\text {rd }}, 2020$. Link titles have been substituted for their associated services in order to make understanding of this data easier.

In the approximate four months since the Wellbeing Check-In service has been live, it has attracted $33.47 \%$ of all the traffic on the SHW hub webpage, making it the most clicked link on the page. When 
this date range is expanded to include the last year, the Wellbeing Check-In service is ranked the second most clicked link on the page, by less than $1 \%$ (see Table 1).

We had anticipated that we would need to spend considerable time and effort to gain traction and become an accessible, common-knowledge support service for the university community. This high percentage result evidenced by these analytics, even in a short timeframe, has exceeded expectations.

\section{Service use $\mathrm{s}^{3}$}

This year, through the Wellbeing Check-In service and the Wellbeing CONNECT programs, the $\mathrm{CCM}$ has coordinated wellbeing responses to a total of 537 cases. Of these, 524 have been through the Wellbeing Check-In service over the four months the program has been live, with the remainder being situations specifically handled by the CCM as a part of their Wellbeing CONNECT program design (see Figure 1).

When we examined the categorised concerns of the Wellbeing Check-In program, overwhelmingly these were the "check-in" calls for which the program was initially designed $(\mathrm{N}=369)$. Although we did see some time variations, on average, this equates to approximately 92 requests per month, over the months the team has been operational. Added to this, the support work that the service began by reaching out to students who demonstrated distress and identified other wellbeing concerns (as a part of their bursary applications), saw the team reach out to an additional 155 students providing comprehensive information summarising the SHW services, outlining multiple resources, and identifying how the students could get in touch if they wanted to access support or resources (see Figure 1).

This would be a significant number of new cases for any of our established support services to manage, however when considering the small staffing allocation for this program, coupled with the significant complexity these cases presented, these case numbers are immense.

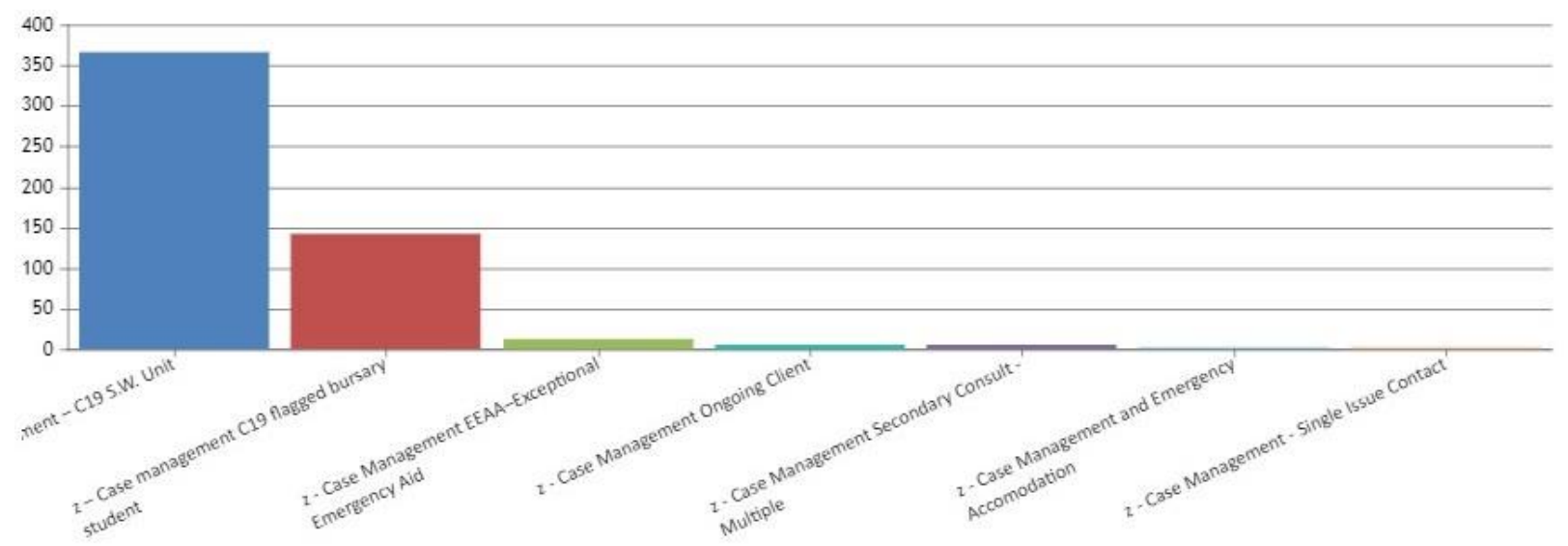

Figure 1 Categorised Concerns of Created Case Files

Note. COVID-19 triage response (369); Flagged bursary students (142); Exceptional Emergency Aid Applicants (13); Ongoing client (5); Multiple secondary consult (5); Case management \& Emergency accommodation (2); Case management single issue contact (1).

There are some definite trends visible when we examine the flow of new cases through the program. Although the service did not go live until the end of March, the dramatic uptake of the service is obvious. While there was a drop in the number of cases through May (although new cases remained

\footnotetext{
${ }^{3}$ Data in this section incorporates information from 1 January $2020-24^{\text {th }}$ July 2020. Although the Wellbeing Check In service did not go live until the end of March, the data range was broadened to encompass the initial crisis response reaction not specified under the Wellbeing Check-In service, as well as to demonstrate the service "pivot".
} 
overwhelmingly high at this point) and more so into June, we attributed this decrease to several factors:

- At this point of the year, all SHW services see a lull in usage due to our students focus on upcoming semester exams.

- Outside the university context, there was also an initial relaxation of the COVID protocols. Given the Check-In service is linked so strongly to the COVID-19 concerns, this seems likely (see Figure 2).

With the shift into the new COVID-19 government restrictions and mandated mask requirements in July, and the end of the university exams period, there was another dramatic increase in the usage of the service (see Figure 2). ${ }^{4}$

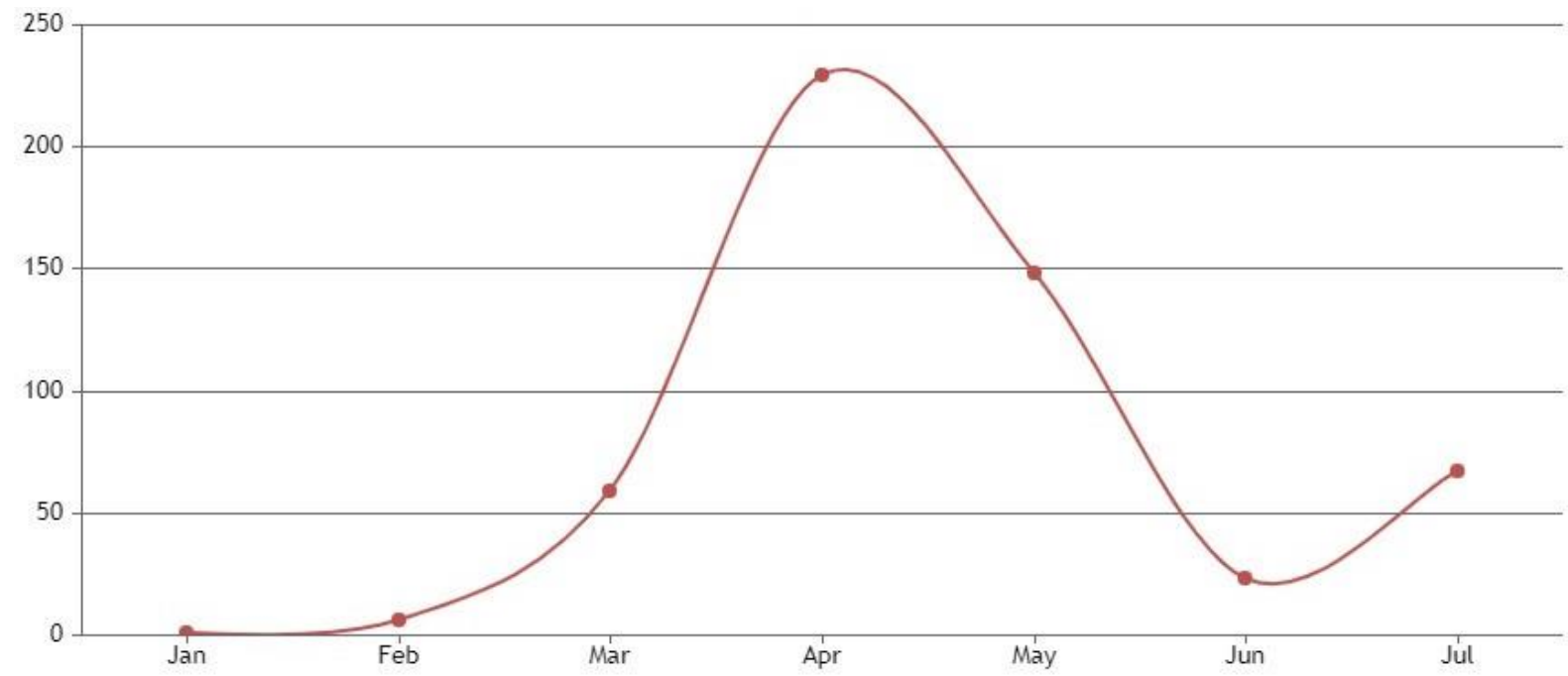

Figure 2 Number of New Created Cases by Month

Note. January (1); February (6); March (59); April (229); May (148); June (23); July (67).

The spread of new cases relative to their flagged concern, is similar to the distribution seen in Figure 2 (see Figure 3). Two main concerns are identifiable, and both echo the same distribution.

The larger of these represents the primary, ongoing work of the team in triaging the first contact from students concerned for their wellbeing because of COVID-19. During April, the peak of our busiest period in the initial state-wide shut down, our number of COVID-19 triaging cases reached 143.

The second distribution represents the team's work in supporting both sets of the financial support application mechanisms through the university. Although a secondary role for the Wellbeing CheckIn program, this focus provided a significant number of cases. Again, at our busiest point in April, the service coordinated outreach to 86 flagged financial bursary applications whose personal statements raised wellbeing concerns.

With the initial financial bursary applications of April and May completed, the Wellbeing Check-In team begun to support exceptional emergency aid applications. It is anticipated that this focus will only increase and has the potential to match the already visible upward trend in triage contacts.

New requests for "ongoing contacts", and "secondary consultation" were marginal during this time and in comparison, barely visible (see Figure 3).

\footnotetext{
${ }^{4}$ Although the data was collected on $24^{\text {th }}$ July in order to facilitate writing and analysis, we feel it noteworthy the final count on new cases created is July was 99 . This figure was not used in any analysis.
} 


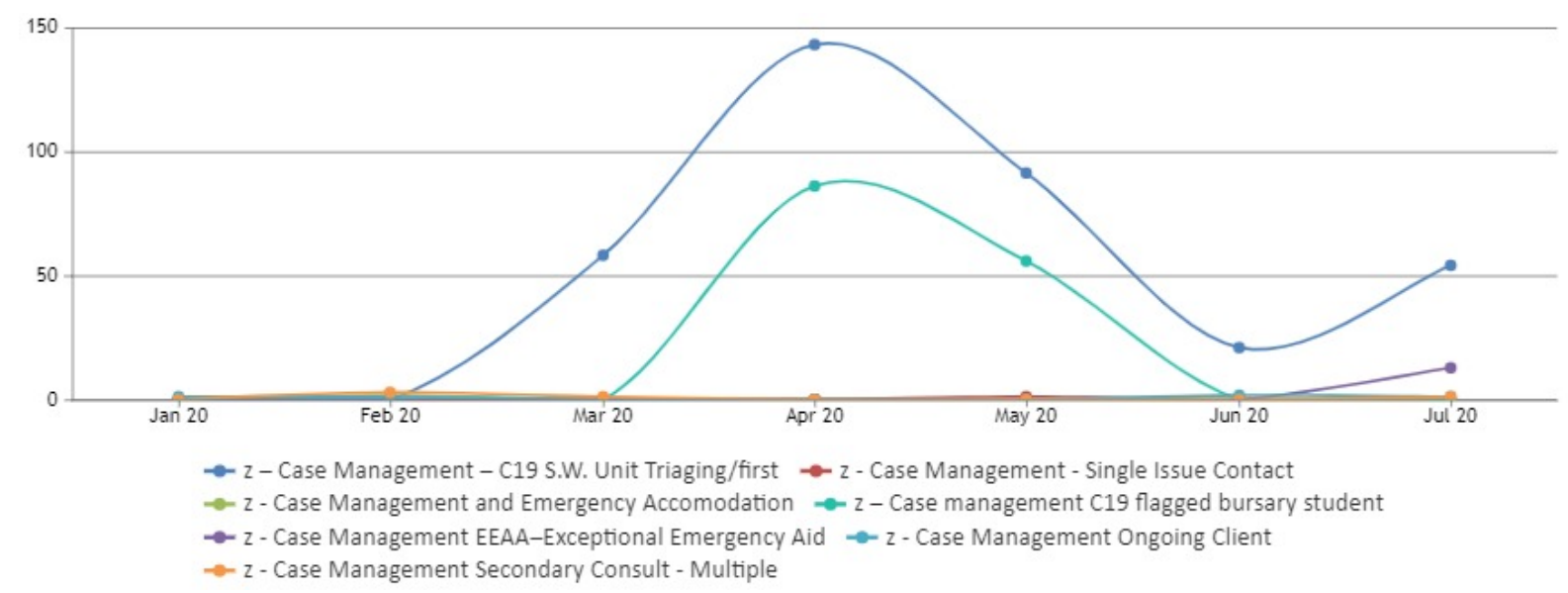

Figure 3 Number of New Created Cases by Month, Separated by Categorised Concerns

Note. January - Ongoing client (1). February - Case management and Emergency accommodation (2); Ongoing client (1); Multiple secondary consult (3). March - COVID-19 triage response (58); Multiple secondary consult (1). April COVID-19 triage response (143); Flagged bursary student (86). May - COVID-19 triage response (91); Case management single issue contact (1); Flagged bursary student (56). June - COVID-19 triage response (21); Ongoing client (2). July - COVID-19 triage response (56); Exceptional Emergency Aid Applicant (13); Ongoing client (1); Multiple secondary consult (1).

The overwhelming majority of referrals coming to the Wellbeing Check-In program came from the Deputy Director of SHW, with almost 160 students accessing the service in this way. It should be noted here that 142 of these referrals were flagged personal statements as a part of the initial financial bursary applications (see Figure 4).

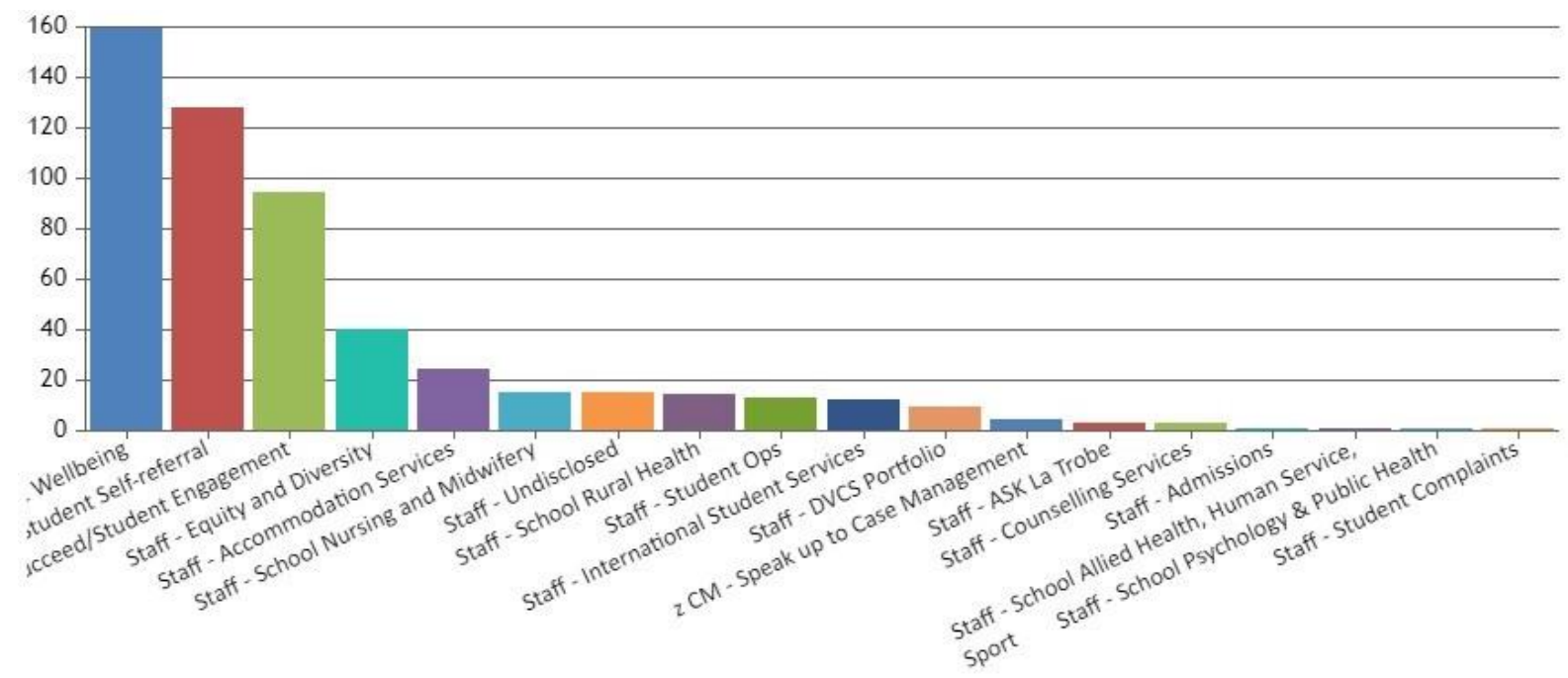

Figure 4 Referring Business Unit of Created Case Files, Including Self-Referral

Note. Student Wellbeing (159); Self-referral (128); Student Success/Engagement (94); Equity \& Diversity (40); Accommodation (24); School of Nursing \& Midwifery (15); Staff - Undisclosed/other (15); School of Rural Health (14); Student Operations (13); La Trobe International (12); DVCS portfolio staff (9); Speak Up to Case Management (4); Ask La Trobe (3); Counselling (3); Admissions (1); School of Allied Health (1); School of Psychology and Public Health 1; Student Complaints (1).

The Wellbeing Check-In team response to students with concerning financial bursary applications was primarily handled administratively by the Project Coordinator. Each student was registered to preserve the context of their situation indicated in their application and were sent a standard packet 
of information on the SHW services, including how to access them remotely. This was to encourage students' self-efficacy and preserve their independence, asking them to get access our supports if they wanted, while holding any of their existing supports paramount. By registering the student, the Wellbeing Check-In team were able to access the application information if the student did reach out for support at a later date.

Because these students were, for the most part, not actively contacted by team Coordinators, and the Wellbeing-Check ins were the primary focus of the program, we have run data assessing referral information solely for the COVID-19 triaging (see Figure 5).

Excitingly, the second most common referrer has been the students themselves $(\mathrm{N}=124)$, with almost $34 \%$ of the team's case work derived from the request of the student. Given this was the specified primary referral pathway that the service aimed to target, to see this reflected in the referral data is a pleasing result.

There has also been a significant number of referrals to the Wellbeing Check-In service as a result of the Student Success Call Out Campaign referrers ( $\mathrm{N}=93$ ). This illustrates that the program has been able to collaborate effectively with other teams in the university to support the wellbeing of our students.

We also saw a significant number of combined referrals $(\mathrm{N}=46)$ coming from the academic staff working with students concerned about, or flagged as, having potential exposures to COVID-19 while on placement.

A further breakdown, explaining some other noteworthy instances of referral (see Figure 5) are as follows:

- Several referrals came from Accommodation Services $(\mathrm{N}=24)$, expressing concern about the wellbeing of students in isolation on-campus.

- Check-in requests from our International Student Services team $(\mathrm{N}=12)$, concerned for the wellbeing of this vulnerable cohort.

- Proactive outreach to 9 students affected by the hard lock down imposed by the Victorian government on Melbourne's public housing high rise towers, to provide targeted wellbeing support. These were flagged by the Portfolio Director.

- An additional 17 referrals were received from the SHW Deputy Director, who identified students from a range of areas as being potentially at elevated risk and warranting a proactive outreach call from the Wellbeing check in team.

Please note: This referral data does not reflect the number of cases where students accessed the service on the recommendation of a staff member as the service database is not programmed to record this intricacy. 


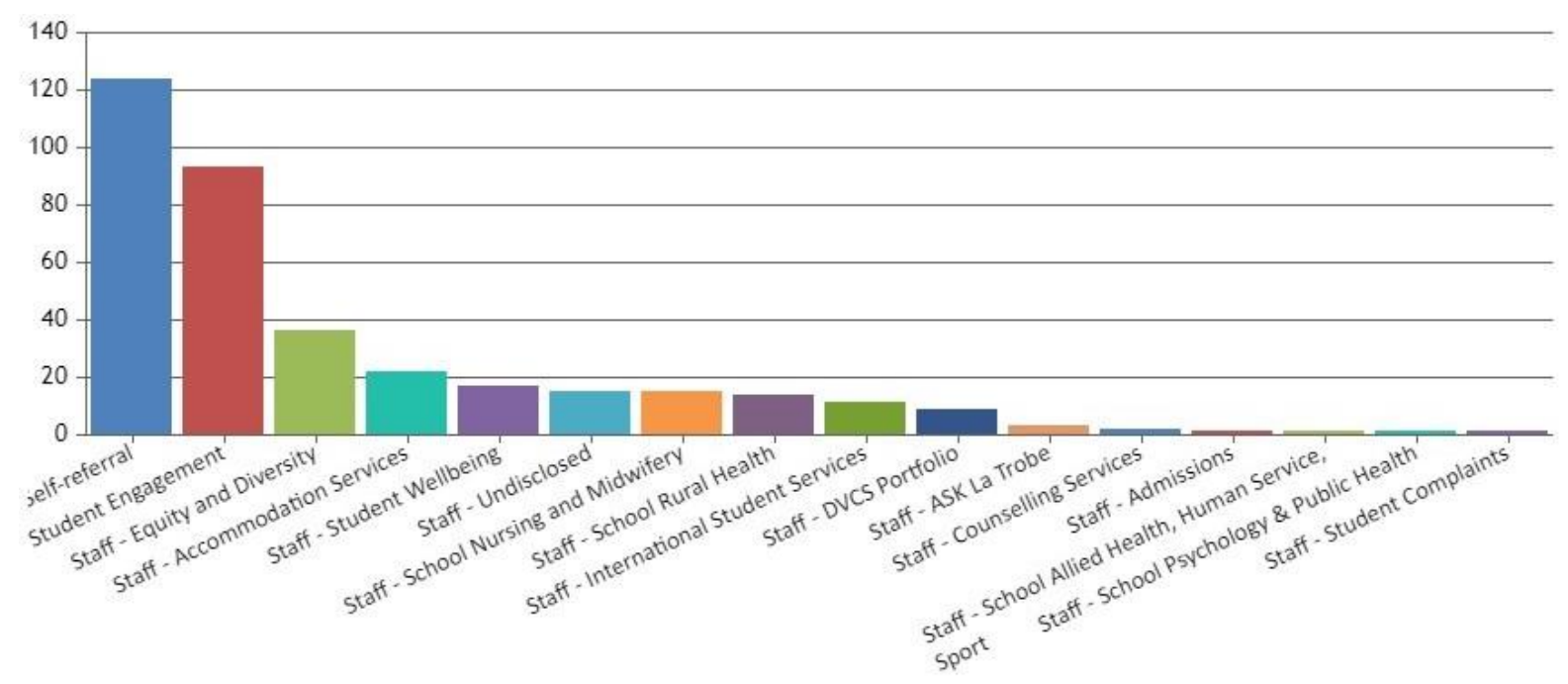

Figure 5 Referring Business Unit of “COVID-19 Triage Response” Case Files, Including Self-Referral

Note. Self-referral (124); Student Success/Engagement (93); Equity \& Diversity (36); Accommodation (22); Student Wellbeing (17); Staff - Undisclosed/other (15); School of Nursing \& Midwifery (15); School of Rural Health (14); La Trobe International (11); DVCS portfolio staff (9); Ask Latrobe (3); Counselling (2); Admissions (1); School of Allied Health (1); School of Psychology and Public Health (1); Student Complaints (1).

We examined the statistics on whether students accessing the Wellbeing Check-In program were domestic or international and identified their primary campus of study.

The Wellbeing Check-In team has, to date, seen almost twice the number of domestic students as international students (see Figure 6). Overwhelmingly, the students' campus of study was Bundoora, being the primary campus of almost $70 \%$ of the program's student contacts (see Figure 7).

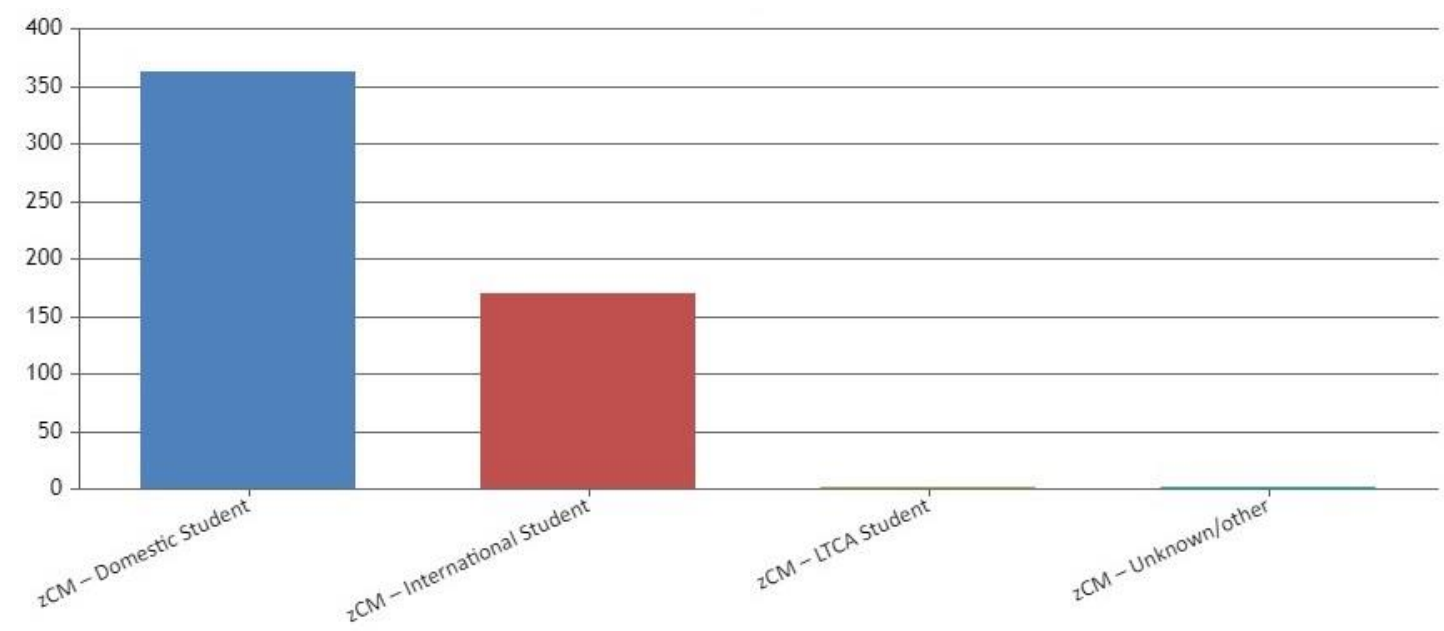

Figure 6 Student Categorisation of Created Case Files

Note. Domestic (363); International (170); LTCA (2); unknown/other (2). 


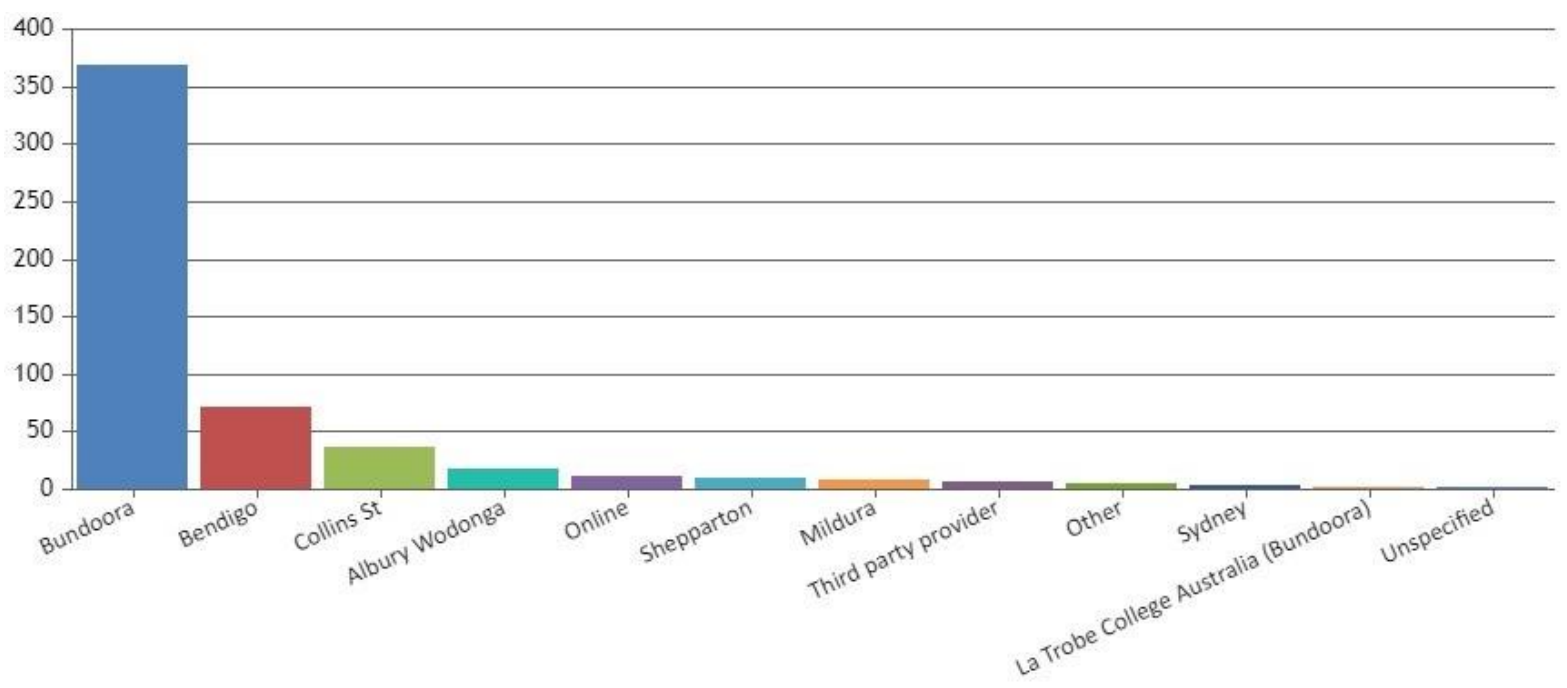

Figure 7 Primary Campus of Created Case Files

Note. Bundoora (368); Bendigo (71); Collins Street/City Campus (36); Albury Wodonga (18); Online (11); Shepparton (9); Mildura (8); Third party provider (6); Other (5); Sydney (3); LTCA (2); Unspecified (1).

As the Wellbeing Check-In program did not formally assess complexity or risk experienced by the students they worked with, the number of file notes attached to cases provided a substitute and approximate measure that could be used to indicate the complexities of the presentations that the team worked with.

Although only an indicator, at the very least the number of file notes used as a measure of complexity demonstrates the time and labour spent in preparing the documentations required for this work. In the time that the Wellbeing Check-In program has been live, the team have created a total of 1,839 file notes (see Figure 8 ). ${ }^{5}$

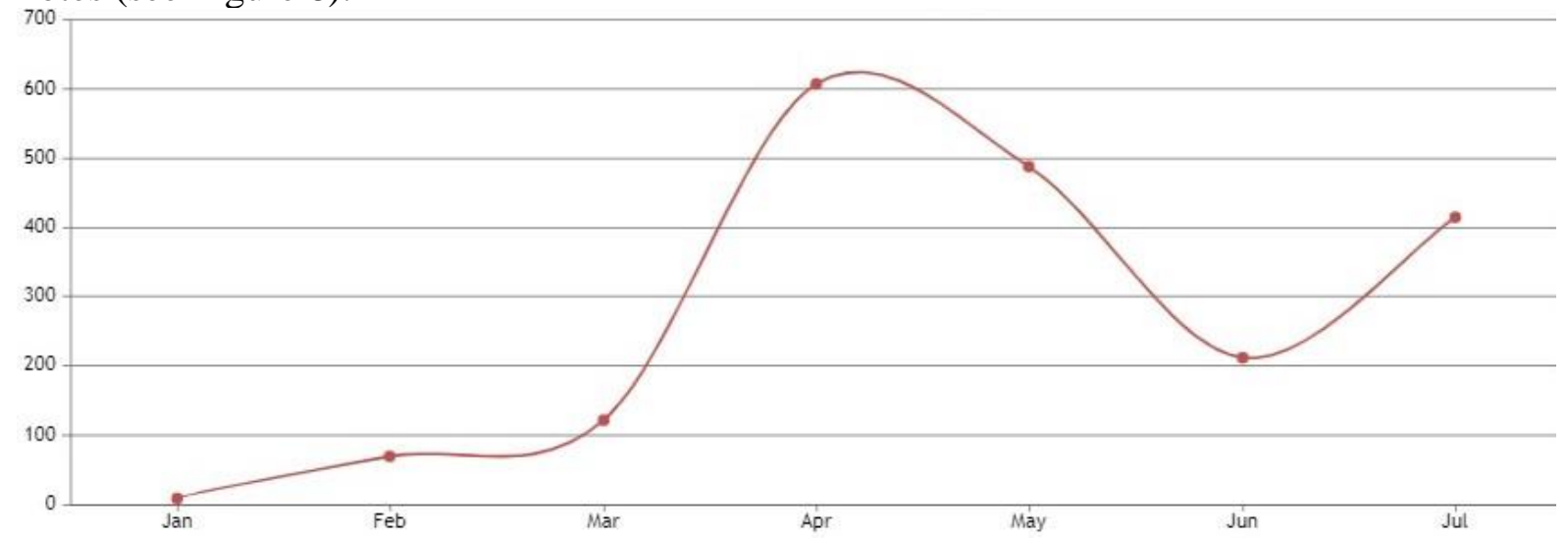

Figure 8 Number of Case Notes Created as Part of the Wellbeing Check-In Program

Note. January (9); February (68); March (122); April (606); May (486); June (212); July (413).

\footnotetext{
${ }^{5}$ Again, Although the data was collected on $24^{\text {th }}$ July in order to facilitate writing and analysis, we feel it noteworthy the final count file notes made in July was 518. This figure was not used in any analysis, nor total note count from the program.
} 


\section{Challenges}

\section{Role and service pivots}

As previously stated, the Wellbeing Check-In service was developed as a bespoke service to support the wellbeing of our students. It responds to the anticipated effects of the COVID-19 pandemic on our students' wellbeing, outside the scope of current SHW service offerings. In contrast, the established Wellbeing CONNECT service provides a smaller number of highly complex students medium- and long-term case management support.

Whilst starting the program with on-board staff members requiring minimal training, this approach did have a cost, albeit not a financial one. By participating in the Wellbeing Check-In initiative, these staff members had less time in which they could complete their normal, day-to-day role functions. This was most evident in the necessitated "pause" to the promotion and building of the CCM's program, "Wellbeing CONNECT". The effect of this new program on the team's ability to focus upon, promote and undertake their normal workload is difficult to define, but important to consider. It is evident that this program has demonstrated a need in our student community for support and intervention that previously was unmet. An investment of additional resources would be welcomed and would allow a further comprehensive, streamlined and intensive response to continue. This is apparent in the data that has been captured.

\section{Operational protocol development}

The Wellbeing Check-In initiative went live quickly, moving from concept to receiving forms and referrals within 48 hours. As a result, a significant amount of work was put into the refinement of the program and service operation, to fine tune the team's processes and protocols, in order to offer a continued and standardised service with the addition of new staff.

Any operational effect on the "continuity" of the program from these refinements seems to only have been on the administration portion of the initiative. The streamlining of referral processes, defining of inbox management and standardising intake procedure was implemented in order to improve work practices and efficiency.

\section{Capturing specific and consistent data}

There was a significant challenge when the program first began to define the types of categorical data that would need to be gathered and recorded for each student. It was important to reconsider what information was required, given the effect this information had on students' eligibility for assistance. Further to this, the database used was bespoke for another SHW team's operational needs, so required adjustment to fit within the pre-determined category fields, in order to meet the Wellbeing Check-In program needs.

As a result, two team members dedicated a significant amount of time to retrospectively code earlier student files in order to accurately represent the scope and reach of the program, and indirectly assess the operational effectiveness of the Wellbeing Check-In service.

\section{University wide “outreach” campaign response and Wellbeing Check-In program refinement}

During six weeks of April and May, the university undertook an outbound, "outreach" call campaign via LTU's Student Success division. During this time, more than 39,961 calls were attempted to 24,360 students, with 13,811 students reached. ${ }^{6}$ Those students who were not able to be reached through a phone call, received an email through the University's Customer Relationship Management System (CRM), providing information on accessing help and resources, including the Wellbeing Check-In service.

\footnotetext{
${ }^{6}$ These figures are accurate as of 11 May 2020, provided in an email from the Manager of Student Success to all participating outreach staff.
} 
The leadership team of Student Success reached out to the CCM before they began their campaign to discuss modes of student referral to the Wellbeing Check-In service, should a caller be concerned for a student's welfare. Outreach callers were asked to refer students they were concerned about to the Student Success leadership, who would then collate and send this information directly to the CCM. Very quickly, the CCM began receiving an unexpectedly high number of requests for a Check-In call. On numerous occasions, the CCM needed to garner support from SHW unit managers to respond to a portion of the requests in order to respond to student needs in a timely manner.

Multiple collaborative conversations with Student Success occurred to streamline the email traffic initially encountered. Agreements were made over time regarding referral format, including a single referral per email, as opposed to multiple referrals in the same email. This allowed smoother logging and triaging of referrals, which in turn enabled the team to clinically assess and respond to urgent referrals and better meet student needs. At the same time, this also ensured record keeping accuracy and workflow efficiency.

Regular dialogue between the CCM and the Student Success team leaders continued throughout the duration of the campaign. The wider SHW team, via the CCM, provided guidance and support by providing and continually refining a possible "script" for callers in the campaign. This script outlined information on the operational and functional scope of each unit in SHW that callers could provide to student in situations that a Wellbeing Check-In was not required, but when students may have wanted to independently access other SHW services. Several students relayed this information as the reason they contacted on their own initiative, various units in SHW including the Wellbeing CheckIn team.

\section{Misplaced referrals and redirected contact}

As the Wellbeing Check-In service progressed and we continued to receive referrals from both students and staff, a number of these were outside the scope of the Wellbeing Check-In service. In general, these were easily redirected to the appropriate SHW unit, such as the Counselling Services, Speak Up or the AccessAbility team.

Some contacts made with the Wellbeing Check-In service were in relation to the financial bursary applications submitted to LTU's university-wide coordinated fund. These were most commonly selfreferral cases received via the website portal. Whilst recognising that financial issues do strongly relate to, and impact on students' wellbeing (and increasing levels of stress), our team were obliged to follow predetermined, mandated guidelines. This required that we advise students that we were unable to respond to their application result via this portal and they needed to follow the direction of the financial bursary protocols provided at their initial application. Despite this, we feel we honoured our own commitment to listen to and respond to these students. We were still able to, at times, refer these students to additional resources and community supports as a result of their contact with us.

The Wellbeing Check-In portal on occasion received detailed and specific queries that related to the COVID-19 State Health Department directives. These students were redirected to the State Health Department's website and in some cases, either the Department of Health and Human Services hotline, or LTU's COVID-19 Frequently Asked Questions webpage. We regularly clarified, and eventually adjusted the introductory text on the service's online request form, to explain that we were not an expert medical service, nor were we able to provide legal directives regarding government protocols.

\section{Conclusions}

The success of the Wellbeing Check-In service has been incredible. With a small team, and a small budget, we have been able to help many students in a very short time and have been able to make a real difference to the university community. 
The impact of COVID-19 and its societal changes on our students' wellbeing cannot be underestimated and it has significantly contributed to an increase in the anxiety and stress of many students.

While often related to self-management and expectations in the "new normal", we have helped our students with significant stressors such as exposure to COVID-19, substantial financial pressures, ongoing or resurfaced mental health concerns, managing new and old anxieties, dealing with isolation and loneliness due to, or exacerbated by the COVID-19 shut-down, and the immense uncertainty of the "new world".

Students also accessed the service as a first point of call because they were concerned about their academic progress and decision making, and many were seeking support for the "first time" because they were impacted by COVID-19 adjustments.

Almost half of the students the Wellbeing Check-In team worked with were international students. These students were in a particularly unique situation in relation to financial matters, limited government support and worry for families in their home country.

The team worked with many first year students, some originating from regional towns, who also demonstrated similar concerns as they went through the multiple adjustments of first coming to the university, then returning home just two weeks later to learn online, being forced to "pause" this anticipated and significant rite of passage.

The Wellbeing Check-In team has provided a number of interventions across the program's operation. This includes, but not limited to;

- Supportive counselling;

- Short term case management;

- Supporting access to and support for exploring resources;

- General education and support around students' feelings of loss of control;

- Perceived or real elevated risk to physical health;

- General self-care education related to nutrition; and

- Hydration and rest, and sharing of resources, or referral to other supports or compiled resources.

Expert commentary and publications have explored the mental health impacts of the COVID-19 pandemic, emphasising that they will be long lasting (Carbone \& Jorm, 2020).

Examples of this include: the recent (May 2020) appointment of a new deputy chief medical officer (Dr Ruth Vine) and the COVID \$48.1 million grant for a National Mental Health and Wellbeing Pandemic Response plan, also released in May 2020. These national campaigns and this appointment "give(s) strong recognition from the government that Australians "are experiencing fear, anxiety, loneliness, financial and family stress as a result of the COVID-19 pandemic and the measures needed to contain it" (Hunt, 2020).

For as long as COVID-19 continues, and the government restrictions remain in place, we anticipate that demand will continue for the Wellbeing Check-In service. Because of this, we are hopeful that the program will continue as it is essential to meeting the requirements our students, filling an identified "gap" in the already existing SHW services.

In this new era of heightened awareness, we are hopeful that we will be able to continue to adjust this program and its format in order to increase the accessibility of support services, reducing clinical associations, and de-stigmatising mental health assistance. 


\section{References}

Australian Association of Social Workers. (2015). Scope of social work practice: Psychosocial assessments. Melbourne: AASW.

Australian Red Cross \& Australian Psychological Society. (2020). Psychological first aid: Supporting people affected by disaster in Australia. Melbourne: Australian Red Cross.

Carbone, S. \& Jorm, A. (2020, April 30). We need to flatten the other coronavirus curve: Our looming mental health crisis. The Conversation. https://theconversation.com/we-need-to-flatten-the-other-coronavirus-curve-ourlooming-mental-health-crisis-137170

Hunt, G. (Minister for Health). (2020, May 16). COVID - 19: \$48.1 Million for National Mental Health and Wellbeing Pandemic Response Plan. Retrieved from https://www.health.gov.au/ministers/the-hon-greg-hunt$\mathrm{mp} / \mathrm{media} /$ covid-19-481-million-for-national-mental-health-and-wellbeing-pandemic-response-plan

Folstein, M.F., Folstein, S.E., \& McHugh, P.R. (1975). Mini-mental state: A practical method for grading the cognitive state of patients for the clinician. Journal of Psychiatric Research 12(3), 189-198

Patrick, J. (2000). Mental status examination rapid record form. Retrieved from https://huibee.com/wordpress/wpcontent/uploads/2013/11/Mental-State-Exam-form.pdf

The authors may be contacted via

s.power@latrobe.edu.au

Please cite this paper as:

Power, S. \& Hanna, G. (2020). The "Wellbeing Check-In" Service Establishing, Developing and Tailoring a New Program to offer a Student-centred Response to COVID-19. Journal of the Australian and New Zealand Student Services Association, 28(2), 80-95. doi.org.10.30688/janzssa.2020.07 